\title{
Optical and evaporative cooling of cesium atoms in the gravito-optical surface trap
}

\author{
M. Hammes, D. Rychtarik, V. Druzhinina, If U. Moslener,fl I. Manek-Hönninger,f and R. Grimm \\ Max-Planck-Institut für Kernphysik, 69029 Heidelberg, Germany
}

(Dated: 02 May 2000)

submitted to Journal of Modern Optics, special issue Fundamentals of Quantum Optics V, edt. by F. Ehlotzky

\begin{abstract}
We report on cooling of an atomic cesium gas closely above an evanescent-wave atom mirror. At high densitities, optical cooling based on inelastic reflections is found to be limited by a densitydependent excess temperature and trap loss due to ultracold collisions involving repulsive molecular states. Nevertheless, very good starting conditions for subsequent evaporative cooling are obtained. Our first evaporation experiments show a temperature reduction from $10 \mu \mathrm{K}$ down to $300 \mathrm{nK}$ along with a gain in phase-space density of almost two orders of magnitude.
\end{abstract}

\section{INTRODUCTION}

Optical dipole traps based on far-detuned laser light have become very popular as versatile tools for the storage of ultracold atomic gases and can be employed for a great variety of experiments, e.g., on quantum phenomena, precision measurements, ultracold collisions and quantum gases [1]. Optical dipole traps have opened up fascinating new experimental possibilities not offered by other trapping methods.

A very important advantage compared with magnetic traps is the fact that a dipole trap can store atoms in any sub-state or mixtures of sub-states of the electronic ground state. Because of the unusual scattering properties of cesium the use of an optical dipole trap may be the only way to achieve Bose-Einstein condensation of this interesting atomic species. For Cs the quantummechanical scattering is resonantly enhanced [2, 3], and binary collisions flipping the spin state lead to severe loss from magnetic traps [4, 5, 6, 7]. The latter effect, which has been explained by resonant scattering in combination with a second-order spin-orbit coupling [8, 9], has so far prevented the attainment of a quantum-degenerate gas of cesium atoms.

Inelastic two-body collisions are energetically suppressed in the absolute internal ground state of cesium, which is the high-field seeking state $F=3, m_{F}=3$. Atoms in this state cannot be trapped magnetically but in an optical trap. In recent experiments, Vuletic et al. have discovered a low-field Feshbach resonance [10, which promises an easy experimental way to tune the $s$ wave scattering length in a wide range. With magnetic fields between $17 \mathrm{G}$ and $30 \mathrm{G}$ a positive scattering length should allow for a stable Bose-Einstein condensate.

In our experiments to explore the quantum gas prop-

*Present address: Physikalisches Institut, Heidelberg University, 69120 Heidelberg, Germany.

${ }^{\dagger}$ Present address: Interdisziplinäres Institut für Umweltökonomie, Heidelberg University, 69115 Heidelberg, Germany.

${ }^{\ddagger}$ Present address: Bordeaux University, 33405 Talence, France. erties of cesium, we use the gravito-optical surface trap (GOST) 11. This optical dipole trap allows one to confine a large sample of atoms in an almost conservative environment with very efficient precooling by a Sisyphustype mechanism. In a second stage, evaporative cooling is implemented to further increase the atomic phase-space density. In this article, we summarize the basic properties of the GOST (Sec. II) and discuss the limitations of optical cooling at high densities (Sec. III). We then report our first evaporative cooling results (Sec. IV]) and discuss the prospects of future experiments (Sec. V).

\section{GRAVITO-OPTICAL SURFACE TRAP}

\section{A. Trapping potential and general properties}

A schematic overview of the geometry of the trap is given in figure 1. The GOST is an "optical mug", whose bottom consists of an evanescent-wave (EW) atom mirror generated by total internal reflection of a blue-detuned laser beam from the surface of a prism, while the walls are formed by an intense hollow beam (HB) which passes vertically through the prism surface.

The steep exponential decay of the EW intensity along the vertical direction and the sharp focussing of the hollow beam lead to large intensity gradients and thus in combination with the blue detuning of both light fields to a strong repulsive dipole force. We exploit this fact to efficiently keep the atoms in the dark inner region of the trap where the unwanted effect of heating through scattering of trapping light photons is suppressed. In addition to that, the concept also features a large trapping volume which allows for a transfer of a large number of atoms into the GOST. Due to the accurate focussing of the hollow laser beam into a ring-shaped intensity profile [12], the shape of the potential is box-like along the horizontal directions whereas the combination of gravity and the repulsive wall of the EW leads to a wedge-shaped potential vertically.

In thermal equilibrium this geometry allows for a simple description of the ensemble. The vertical density pro- 


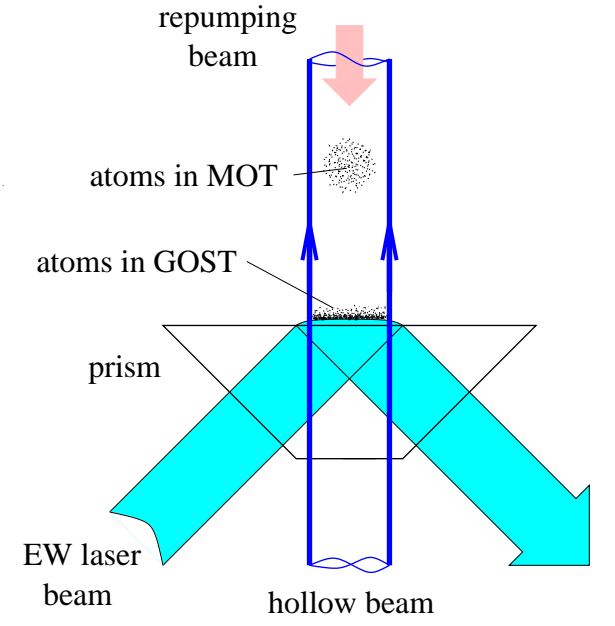

FIG. 1: Illustration of the gravito-optical surface trap.

file is given by the "barometric" equation

$$
n(z)=n_{0} \exp \left(-z / z_{0}\right)
$$

with $n_{0}$ being the peak number density and $z_{0}=$ $k_{B} T / m g$ the $1 / e$-height of the sample. $m$ denotes the mass of the cesium atom, $g$ the gravitational acceleration, $z$ the vertical coordinate and $T$ the temperature. Assuming a homogeneous horizontal density distribution within the hollow beam radius $r_{H B}$ this expression can be integrated to express the peak density $n_{0}$ in terms of the atom number $N$ and the temperature $T$,

$$
n_{0}=\frac{m g}{\pi r_{H B}^{2} k_{B}} \frac{N}{T}
$$

The mean density $\bar{n}$ defined as

$$
\bar{n} \equiv \frac{1}{N} \int d^{3} r n^{2}(\mathbf{r})
$$

is exactly half of the peak density $\left(\bar{n}=n_{0} / 2\right)$. In the GOST potential the scaling of density with temperature $\left(n \propto T^{-1}\right)$ is somewhat less as compared to a 3D harmonic oscillator where $n \propto T^{-3 / 2}$.

The mean energy per atom is $\bar{E}=5 / 2 k_{B} T$, of which $3 / 2 k_{B} T$ are kinetic energy and one $k_{B} T$ potential energy in the vertical gravitational field, as can be obtained using the Virial theorem. In the horizontal degree of freedom the box form of the confining potential leads to negligible potential energy.

While the horizontal extension of the sample is determined by the diameter of the hollow beam $\left(2 r_{H B}\right)$, the height is proportional to the ensemble temperature with a constant of proportionality of $6.4 \mu \mathrm{m} / \mu \mathrm{K}$. At typical equilibrium temperatures of a few $\mu \mathrm{K}$ the $1 / e$-height is on the order of a few ten $\mu \mathrm{m}$ thus leading to a highly anisotropic "pancake-shape" of the ensemble.

\section{B. Experimental realization}

The experimental constituents of the GOST are the evanescent-wave diode laser, a titanium:sapphire laser to create the hollow beam and an additional diode laser to provide the light for the repumping beam. For EW and repumping beam we use laser diodes (SDL-5712-H1, distributed Bragg reflector), which yield up to $100 \mathrm{~mW}$ of radiation at the cesium $\mathrm{D}_{2}$-line at a wavelength of $852 \mathrm{~nm}$.

The EW laser is focussed to provide a round spot on the surface of the prism with a $1 / e^{2}$-radius of $540 \mu \mathrm{m}$. The angle of incidence is $\theta=45.6^{\circ}$ which is $2^{\circ}$ above the critical angle of $43.6^{\circ}$. This leads to a $1 / e^{2}$ decay length of $\Lambda=(\lambda / 2 \pi)\left(n^{2} \sin ^{2} \theta-1\right)^{-1 / 2} \approx 500 \mathrm{~nm}$, where $n=1.45$ is the refractive index of the fused-silica prism.

The EW detuning $\delta_{E W}$ is between a few $\mathrm{GHz}$ during optical Sisyphus cooling and up to $300 \mathrm{GHz}$ in the process of evaporative cooling. Since atoms are predominantly kept in the lower hyperfine ground state $(F=3)$ in the dipole trap, all detunings given throughout this paper refer to the transition between this state and the center of the ${ }^{2} \mathrm{P}_{3 / 2}$ excited-state manifold.

At $\delta_{E W} / 2 \pi=3 \mathrm{GHz}$ and a power of $45 \mathrm{~mW}$ the EW creates a repulsive optical potential with a height of $\sim 1 \mathrm{mK}$. The potential barrier is reduced by typically a factor of two by the attractive van-der-Waals interaction between the atoms and the dielectric surface [13].

The hollow beam is generated using an axicon optics 12 to create a ring-shaped focus of an inner and outer 1/e-radius of $r_{H B}=260 \mu \mathrm{m}$ and $r_{H B}+\Delta r_{H B}=290 \mu \mathrm{m}$, respectively. It has a power of $350 \mathrm{~mW}$ and its detuning is in the range between $-0.3 \mathrm{~nm}$ and $-2 \mathrm{~nm}$. The HB provides a potential barrier on the order of $100 \mu \mathrm{K}$ height. At this detuning the HB potential is almost conservative as the photon scattering rates can be estimated to be on the order of a few photons up to a few ten photons per second.

The repumping beam needed for the optical Sisyphus cooling [14] is resonant with the $F=4 \rightarrow F^{\prime}=4$ hyperfine transition of the $D_{2}$-line and has an intensity on the order of $1 \mu \mathrm{W} / \mathrm{cm}^{2}$. It is shone on the trapping region from above (see fig. 11).

\section{Loading of the trap}

The loading scheme of the GOST goes along standard ways starting from an effusive atomic beam which is decelerated by a Zeeman slower [15]. A magneto-optical trap (MOT) collects about $3 \times 10^{8}$ atoms under ultrahigh vacuum conditions $\left(\sim 10^{-11}\right.$ mbar $)$. The sample is then cooled to $\sim 10 \mu \mathrm{K}$ and spatially compressed using a polarization gradient cooling scheme, in which the MOT laser detuning is increased within $50 \mathrm{~ms}$ from $3 \Gamma$ to $14 \Gamma$; here $\Gamma / 2 \pi=5.3 \mathrm{MHz}$ denotes the natural linewidth. After the atomic cloud is shifted from its loading position ( $3 \mathrm{~mm}$ above the surface) to a release position $(0.5 \mathrm{~mm})$ close to the evanescent wave using magnetic offset fields, 
the MOT-laser beams are shuttered and the atoms fall onto the EW-light sheet. As soon as the near-resonant light fields of the MOT are switched off, the repumping beam of the GOST is turned on to optically pump the atoms into the lower hyperfine state $(F=3)$, and the Sisyphus cooling in the GOST starts. Initially up to $2 \times 10^{7}$ atoms are transfered into the dipole trap and undergo optical cooling.

The number of atoms $N$ remaining in the GOST after a variable storage time is measured by recapturing them into the MOT and taking a fluorescence picture using a slow-scan CCD camera. The integrated fluorescence signal, calibrated with a more accurate absorption image of the MOT, allows us to determine $N$ with an estimated uncertainty of less than $50 \%$.

\section{OPTICAL COOLING AT HIGH DENSITIES}

Here we discuss the efficient EW Sisyphus cooling mechanism and its role as optical precooling stage for evaporative cooling in the GOST. We present our observations of density-dependent effects that limit EW cooling, namely an excess temperature and trap loss due to ultracold collisions in the presence of blue-detuned light.

\section{A. Evanescent-wave Sisyphus cooling}

The optical cooling process is based on inelastic reflections of the repeatedly bouncing cesium atoms from the evanescent wave [14, 16]. In the great majority of the bounces the atoms are coherently reflected in the lower hyperfine state $(F=3)$ without any dissipation of kinetic energy or heating. However, occasionally the absorption of an EW photon takes place and the subsequent spontaneous decay will either have the atom end up in the $F=3$ - or the $F=4$ hyperfine state. A decay into the upper hyperfine state occurs with a branching ratio of $q=$ 0.25 and due to the reduced dipole force on this state will lead to a weaker repulsion of the atom from the EW and thereby to a damping of the vertical motion. After leaving the EW the atom is optically pumped back into the lower hyperfine state. Simple considerations on the balance of cooling through this process and heating due to photon scattering lead to an expression for the equilibrium temperature:

$$
T=\left(\frac{1}{q}+\frac{1}{q_{\mathrm{r}}}\right)\left(1+\frac{\delta_{\mathrm{EW}}}{\delta_{\mathrm{HFS}}}\right) T_{\mathrm{rec}} .
$$

The first term in brackets represents the average number of photons scattered per cooling reflection. $q_{r}=5 / 12$ denotes the branching ratio of the decay of the $F^{\prime}=4$ excited state into the $F=3$ ground state. $\delta_{\mathrm{EW}}$ is the detuning of the evanescent wave and $\delta_{\mathrm{HFS}}=2 \pi \times 9.2 \mathrm{GHz}$ the hyperfine splitting of the ground state. $T_{\mathrm{rec}}=200 \mathrm{nK}$ is the recoil temperature of cesium. The experimental parameters yield a cooling limit of slightly less than $2 \mu \mathrm{K}$.

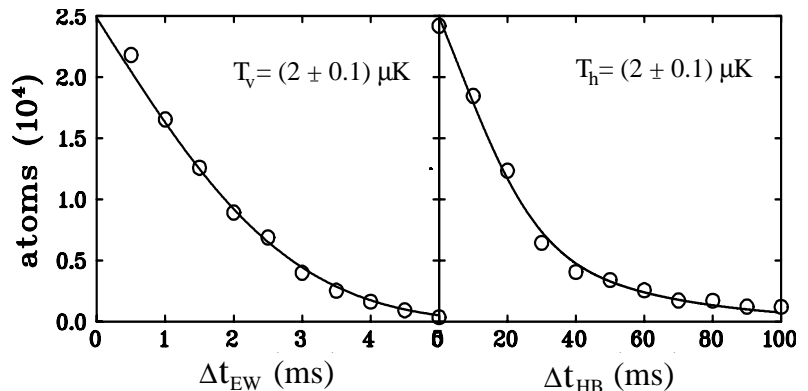

FIG. 2: Measurement of the equilibrium temperature for $2.5 \times 10^{4}$ trapped atoms at a large HB detuning of $2 \mathrm{~nm}$. The employed release-and-recapture method allows to determine the horizontal and vertical temperatures $T_{h}$ and $T_{v}$ separately. This is accomplished by switching off either the EW or the $\mathrm{HB}$ for a short time interval $\Delta t_{E W}$ or $\Delta t_{E W}$, respectively, and measuring the corresponding loss of atoms. Under equilibrium conditions the sample has an isotropic temperature, which in this case $(T=2.0 \mu \mathrm{K})$ is very close to the theoretical cooling limit of EW Sisyphus cooling.

These considerations are consistent with observed temperatures of $2.0 \mu \mathrm{K}$ in small samples $\left(N \lesssim 10^{5}\right.$ atoms) at large hollow beam detunings (see figure 2). Dense samples with $N \gg 10^{5}$ atoms show an excess temperature as will be discussed in the following section.

The time scale on which the cooling process takes place is on the order of one second - considerably longer than in a MOT. Theoretical considerations on the cooling dynamics 14 predict an exponential drop of the vertical temperature in the beginning of the cooling process with a rate constant

$$
\beta=\frac{q}{3} \frac{\delta_{H F S}}{\delta_{E W}} \frac{m g \Lambda}{\hbar\left(\delta_{E W}+\delta_{H F S}\right)} \Gamma .
$$

At $\delta_{E W}=2 \pi \times 3 \mathrm{GHz}$ we find $\beta=1.1 \mathrm{~s}^{-1}$.

Cooling of the horizontal motion is facilitated through mixing of the vertical and the horizontal degrees of freedom by either diffuse reflection of atoms from the EW [11, 17] or in the case of dense samples by elastic collisions. Right after the transfer from the MOT into the GOST, the potential energy from the fall is gradually converted into thermal energy and leads to a very hot $(\sim 100 \mu \mathrm{K})$ sample after thermalization within $\sim 0.5 \mathrm{~s}$. The few seconds it takes for the ensemble to reach the equilibrium temperature are consistent with the calculated cooling rate.

To measure temperatures we use a release-andrecapture method which is accomplished by turning off the EW-potential for a short duration (few milliseconds) and measuring the remaining fraction of atoms as a function of this release time. A fit of a theoretical model to the data which is based on a Boltzmann distribution [15], yields $T$ as the only fit parameter. Under the conditions of the experiments reported here the sample is almost thermalized at any time, so that separate mea- 


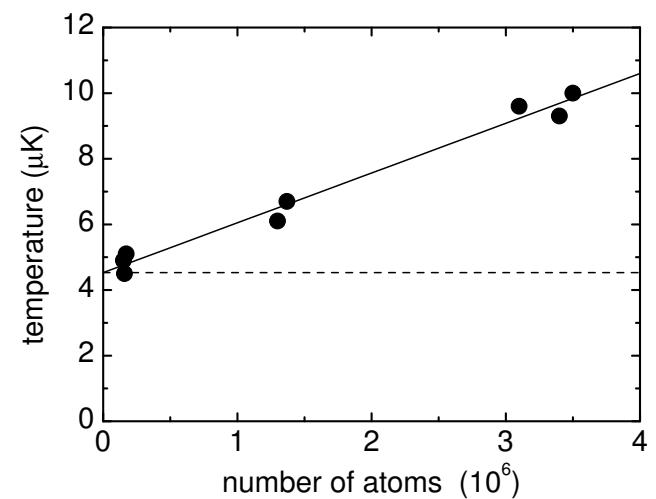

FIG. 3: Equilibrium temperature versus atom number for $\delta_{E W} / 2 \pi=6 \mathrm{GHz}$ and a HB detuning of $0.3 \mathrm{~nm}$. The solid line represents a linear fit to the experimental data (dots). The dashed line indicates the resulting limit temperature for very low densities.

surements of the horizontal temperature were not routinely performed.

\section{B. Excess temperature in dense samples}

Measurements of $T$ in large atomic samples yield significantly higher temperatures $(\sim 10 \mu \mathrm{K})$ compared to what we found in earlier experiments on small samples [11. It turns out that the equilibrium temperature of $\mathrm{EW}$ Sisyphus cooling depends critically on the number of trapped atoms. Figure 3 shows the strong dependence of $T$ on atom number for typical operating conditions of the GOST. The data is reasonably described by a linear dependence

$$
T=T_{0}+a N,
$$

where $T_{0}=4.5 \mu \mathrm{K}$ is the limit temperature achieved in small samples and $a=1.5 \mu \mathrm{K} /\left(10^{6}\right.$ atoms $)$ the slope. The limit temperature $T_{0}$ can be reduced close to the EW Sisyphus cooling limit (see eq. (1) by increasing the detuning of the hollow beam (see figure 2), which is easily explained by heating due to photon scattering. However at large detunings it is not possible to transfer large numbers of atoms into the GOST so that one has to find a compromise between appreciable atom number and low temperature.

The excess temperature as observed in large samples indicates the presence of multiple photon scattering. Similar observations are made in experiments on "gray molasses" where one also keeps the atoms predominantly in the $F=3$ ground state $[18$. We find that the slope $a$ decreases with increasing $\mathrm{HB}$ detuning. It is however not obvious that this excess heating solely depends on the hollow beam. The repumping beam of EW Sisyphus cooling is likely to contribute to this effect.
In a new experimental setup, in which the HB optics was substantially improved to provide aberration-free focussing 19, we are now able to trap $10^{7}$ atoms at a HB detuning of $1 \mathrm{~nm}$ and achieve a temperature of $\sim 10 \mu \mathrm{K}$. This is better by a roughly a factor of two as compared to the conditions of the reported experiments. The improved setup was used for the evaporation experiments in Sec. IV.

\section{Binary collisions in a blue-detuned light field}

Lifetimes of samples of more than $10^{6}$ atoms were found to be on the order of 5 to 10 seconds and clearly indicated the presence of a strong nonexponential contribution to the decay (see figure III C). We model the decay assuming the presence of a two-body loss process in the standard loss rate equation 20

$$
\dot{N}=-\alpha N-\beta \bar{n} N \text {. }
$$

To solve this equation one has to consider the complete dependence of the mean density $\bar{n}$ on the atom number $N$, which is also influenced by the observed excess temperature. Since cooling and thermalization takes place on a time scale much shorter than the decay one can assume stationary conditions for $T(N)$ according to eq. 6 . Using this and equation 2 one obtains

$$
\bar{n}=\frac{m g}{2 \pi r_{H B}^{2} k_{B}} \frac{N}{T_{0}+a N} .
$$

Solving the differential equation one can almost perfectly fit the experimental data and extract the quadratic loss coefficient $\beta$ as a fit parameter. Figure III demonstrates the good agreement between the model and the measurement. We find $\beta$ to be on the order of $10^{-12} \mathrm{~cm}^{3} / \mathrm{s}$, which is about an order of magnitude less as compared to radiative escape in a MOT [20].

A possible mechanism for the observed loss is based on the excitation of a pair of colliding cesium atoms into a repulsive molecular state [21, 22, 23, 24]. The resonant dipole-dipole interaction splits the excited molecular state into an attractive and a repulsive branch. While the attractive branch gives rise to loss processes like radiative escape and photoassociation in red-detuned light, the repulsive branch becomes relevant in blue-detuned light. When a cesium pair is excited into a repulsive molecular state at the classical Condon point it is accelerated along the potential curve and obtains a kinetic energy equivalent to the detuning of the exciting light field with respect to the atomic transition. For a dipole trap the resulting energy gain is in general much larger than the trap depth and leads to an ejection of both colliding atoms from the trap. According to a simple semi-classical model the corresponding rate coefficient $\beta$ should scale with the laser detuning and intensity as $I / \delta^{2}$ as long as the intensity is low enough to avoid optical shielding effects [21]. 


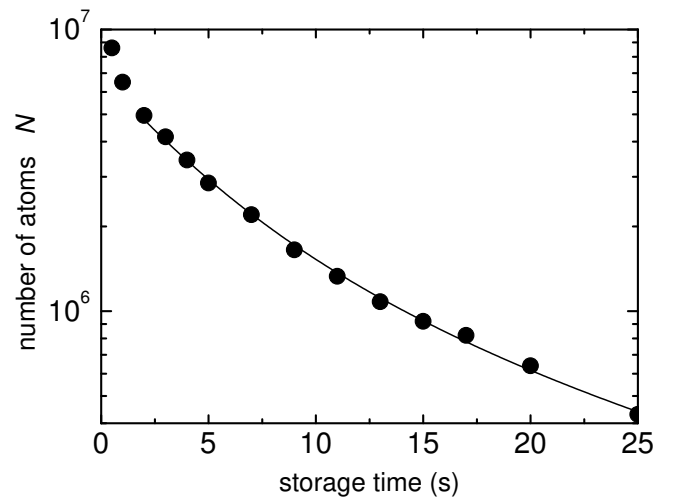

FIG. 4: Decay of the number of trapped atoms with increasing storage time under the same experimental conditions as in fig. 3. The measured data points (dots) are well fitted by a theoretical model that takes into account loss due to binary collisions. The fit (solid line) yields $\alpha=0.03 \mathrm{~s}^{-1}$ and $\beta=$ $0.9 \times 10^{-12} \mathrm{~cm}^{3} / \mathrm{s}$ for the rate coefficients.

In a first set of measurements to investigate this loss process we obtained decay curves at different detunings of the EW-laser field and extracted the rate coefficient $\beta$. In a gravito-optical trap the mean EW dipole potential experienced by the bouncing atoms is given by $\bar{U}_{d i p}=m g \Lambda / 2$, independent of intensity and detuning. Therefore the mean intensity $\bar{I}$ to which an atom is exposed is proportional to the detuning $\delta_{E W}$. Thus the expected scaling of the rate coefficient is $\beta \propto$ $\bar{I} / \delta_{E W}^{2} \propto 1 / \delta_{E W}$. Measurements in the detuning range between $1 \mathrm{GHz}$ and $7 \mathrm{GHz}$ 25] indeed showed the expected $1 / \delta_{E W}$-behaviour. This confirms that collisions in the evanescent wave can explain the observed loss.

As a practical consequence of this fact we can minimize trap loss during optical cooling by switching the EW detuning to higher values shortly after loading. Only in the initial cooling phase low detuning is required to provide a sufficiently large potential barrier. Therefore, in experiments aiming at high densities, the detuning is routinely switched from $3 \mathrm{GHz}$ to about $7 \mathrm{GHz}$ after $0.5 \mathrm{~s}$.

To prove that excitation into repulsive molecular states leads to trap loss we use an additional blue-detuned "catalysis" laser to induce collisional trap loss. In this way this process can be studied independently of the parameters of the trapping potential. The rate coefficient $\beta_{\text {cat }}$ was measured for different values of detuning and intensity of the catalysis laser. In additional temperature measurements we ensured that heating due to photon scattering from the catalysis laser was negligible for all combinations of intensity and detuning. Data was collected at different catalysis laser intensities. The results for $\beta_{\text {cat }}$ shown in figure 5 agree with the expected scaling behaviour $I / \delta^{2}$.

As an important conclusion of these results, trap loss by binary collisions involving repulsive excited molecular

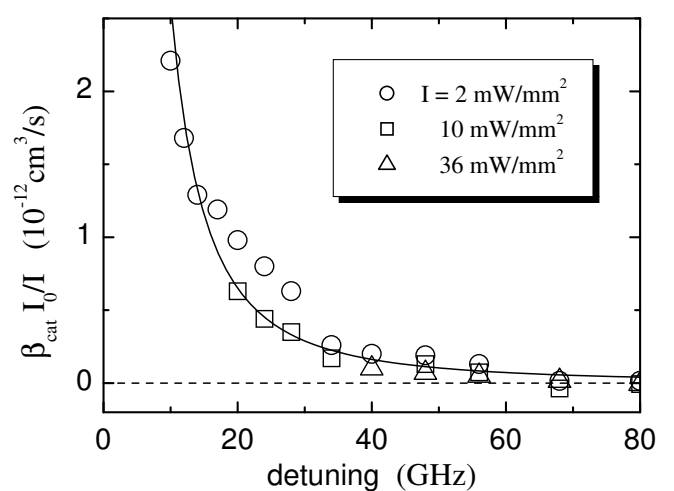

FIG. 5: Rate coefficient $\beta_{\text {cat }}$ describing the loss induced by an additional, blue-detuned "catalysis" laser. The experimental data are taken for three different intensities and, in order to check the expected proportionality to $I / \delta^{2}$, are scaled to the lowest intensity $I_{0}=2 \mathrm{~mW} / \mathrm{cm}^{2}$. The resulting $1 / \delta^{2}$-behavior is indicated by the solid fit curve. The deviations of some data around $20 \mathrm{GHz}$ is likely due to drifts of the experimental conditions.

states plays a significant role in optical cooling. Nevertheless, a dense sample of many atoms at high densities can prepared. If the evanescent wave is then operated at very large detunings, a conservative trapping potential is obtained with very low photon scattering and strongly suppressed trap loss, i.e. a very good starting point for evaporative cooling.

\section{EVAPORATIVE COOLING}

The GOST offers favorable conditions to implement forced evaporative cooling 26 with the prospect to attain quantum-degeneracy of cesium or a two-dimensional quantum gas. In contrast to red-detuned dipole traps used for evaporation experiments [27, 28], the spatial compression of the cold sample essentially results from gravity and is thus not affected when the optical potentials are ramped down. Moreover, many more atoms are initially loaded into the GOST as compared to typical red-detuned traps. Here we describe our first experiments demonstrating the feasibility of efficient evaporation in the GOST.

In our evaporation experiments we operate the trap at a HB detuning of $-1 \mathrm{~nm}$ and keep the EW parameters as in the measurements described above. In two seconds of optical cooling we prepare $N=10^{7}$ atoms at a temperature of $T=10 \mu \mathrm{K}$, and a peak density of $n_{0}=$ $6 \times 10^{11} \mathrm{~cm}^{-3}$. For the unpolarized sample in the sevenfold degenerate $F=3$ ground state this corresponds to a peak phase-space density of $D=n_{0} \lambda_{d B}^{3} / 7 \approx 10^{-5}$ where $\lambda_{d B}=h / \sqrt{2 \pi m k_{B} T}$ is the thermal de-Broglie wavelength. Elastic collisions take place at a rate on 

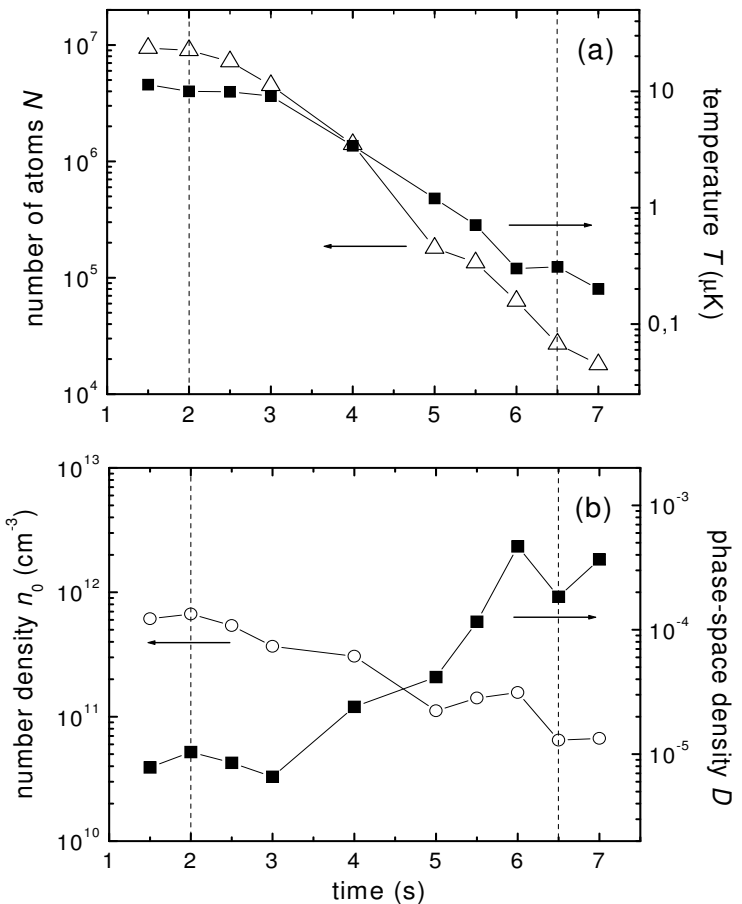

FIG. 6: Evaporative cooling in the GOST. The detuning ramp of the EW starts after two seconds of optical cooling and ends $4.5 \mathrm{~s}$ later, as indicated by the vertical dashed lines. The evolution of the number $N$ and the temperature $T$ of the trapped atoms is shown in (a), the corresponding behavior of peak density $n_{0} \propto N / T$ and phase-space density $D \propto N / T^{5 / 2}$ is displayed in (b).

the order of $50 \mathrm{~s}^{-1}$ and, considering the resonant scattering of cesium [2], lead to a thermalization time of about $200 \mathrm{~ms}$.

To implement forced evaporation we lower the EW potential by ramping up the EW detuning. This simultaneously reduces heating due to photon scattering and suppresses loss through the collision mechanism described above. Within 4.5 seconds the EW detuning is increased exponentially from initially $7 \mathrm{GHz}$ up to $250 \mathrm{GHz}$. This is accomplished by rapid mode-hop free temperature tuning of the EW diode laser. In the last 2.5 seconds of the evaporation ramp the intensity of the hollow beam is reduced from $350 \mathrm{~mW}$ to $11 \mathrm{~mW}$ in order to reduce possible heating by residual light in the dark center of the hollow beam. The contribution of the HB potential ramp to the evaporation remains very small.

The experimental results are shown in figure 6. About $1 \mathrm{~s}$ after starting the exponential ramp, the temperature begins to drop [filled squares in (a)]. At the end of the ramp, it has reached $T \approx 300 \mathrm{nK}$. This decrease of $T$ by about 1.5 orders of magnitude is accompanied by a decrease of the particle number $N$ [open triangles in (a)] from $10^{7}$ down to $\sim 3 \times 10^{4}$, i.e. about 2.5 orders of mag- nitude.

Although the number density $n_{0} \propto N / T$ [open circles in (b)] decreases by about one order of magnitude, the phase-space density $D \propto n_{0} T^{-3 / 2} \propto N T^{-5 / 2}$ [filled squares in (b)] shows a substantial increase by 1.5 orders of magnitude. At the end of the ramp, we obtain a phase-space density of $\sim 3 \times 10^{-4}$.

In the regime of resonant elastic scattering $(T \gtrsim 1 \mu \mathrm{K}$ [3]), the relevant cross section scales $\propto T^{-1}$. In the GOST potential, this leads to a scaling of the elastic scattering rate and the thermal relaxation rate $\propto N T^{-3 / 2}$. Therefore the elastic scattering rate is almost constant for the conditions of our experiments. However, no runaway regime is reached.

An obvious problem in our evaporation scheme is that, for the applied exponential ramp, it takes about one second until the EW potential barrier becomes low enough to start the evaporation. Up to this point already about $50 \%$ of the particles are lost, presumably by the collisional mechanism investigated in Sec. III d. After the corresponding initial loss of phase-space density the later evaporation then leads to a gain of almost two orders of magnitude. This clearly shows that the potential of evaporative cooling in the GOST is much larger than we could demonstrate in our first experiments discussed here.

Although the reported evaporation results are still quite preliminary they already show that efficient evaporation is attainable in the GOST by ramping down the optical trapping potential. Substantial improvements can be expected by an optimized evaporation ramp of the EW in combination with a simultaneous evaporation through the hollow-beam potential.

\section{CONCLUSIONS AND OUTLOOK}

We have investigated optical and evaporative cooling in the gravito-optical surface trap. At high densities, optical cooling by inelastic reflections from the evanescent-wave bottom of the trap was found to be limited by an excess temperature, which we interpret as a result of multiple photon scattering. In addition, a loss process is induced by the blue-detuned trap light. Colliding atoms are excited into a repulsive molecular state which is followed by an energy release into the relative motion. Nevertheless, very good starting conditions are obtained for evaporative cooling. By reducing the trapping potentials we have cooled the sample down to a temperature of $300 \mathrm{nK}$ and obtained a phase-space of $3 \times 10^{-4}$.

So far all our evaporation experiments have been performed with unpolarized atoms in the seven-fold degenerate $F=3$ ground state. Dramatic improvements can be expected by polarizing the atoms into the absolute ground state $F=3, m_{F}=3$. The phase-space density can be increased by a factor of seven, and by producing identical bosons the elastic collsion rate goes up by nearly a factor of two. Moreover, Feshbach tuning can be applied to modify the $s$-wave scattering length [10]. 
We have already performed first experiments, in which we have demonstrated that atoms the GOST can be optically pumped into the state of interest 29].

The spatial compression of the atomic gas can be enhanced by adding a far red-detuned laser beam to the GOST. If such a beam propagates vertically in the center of the hollow beam it provides an additional potential well for transverse confinement. As an example, a 500$\mathrm{mW}$ beam from a compact Nd:YAG laser focused to a 1/e-diameter of $0.1 \mathrm{~mm}$ already provides a dipole potential $U_{\text {red }}$ of $\sim 3 \mu \mathrm{K}$ depth. In the GOST such an additional well can be loaded by elastic collisions, which in thermal equilibrium would result in a peak density enhancement of $\exp \left(\left|U_{\text {red }}\right| / k_{B} T\right)$ along with a corresponding increase in phase-space density 30].

Another interesting option with an additional far reddetuned laser beam is to create a double-EW trap as suggested in Ref. [31. The combination of a repulsive blue-detuned $\mathrm{EW}$ in combination with an attractive reddetuned EW of much larger decay length would allow to create a wavelength-size potential well very close to the dielectric surface. In such a scheme, a situation can be realized where only one vertical bound state exist. The situation would then be similar to atomic hydrogen on liquid helium, for which a two-dimensional quantum gas has been reported [32]. The realization of such a system with alkali atoms could provide much more insight into the physical behavior of such a $2 \mathrm{D}$ gas.

In addition to such experiments on quantum gas properties, the GOST also represents a very promising source of ultracold atoms for experiments related to, e.g., atom interferometry [33], atom-surface interactions [34, 35, and quantum chaos [36].

\section{Acknowledgments}

This work was supported by the Deutsche Forschungsgemeinschaft in the frame of the Gerhard-HessProgramm. We thank D. Schwalm for continuous support and encouragement. One of us (V.D.) acknowledges a fellowship by the Konrad-Adenauer-Stiftung.
[1] R. Grimm, M. Weidemüller, and Yu.B. Ovchinnikov, Adv. At. Mol. Opt. Phys. 42, 95 (2000).

[2] M. Arndt, M. Ben Dahan, D. Guéry-Odelin, M. Reynolds, and J. Dalibard, Phys. Rev. Lett. 79, 625 (1997).

[3] S.A. Hopkins, S. Webster, J. Arlt, P. Bance, S. Cornish, O. Marago, and C.J. Foot, Phys. Rev. A 61, 032707 (2000).

[4] J. Söding, D. Guéry-Odelin, P. Desbiolles, G. Ferrari, and J. Dalibard, Phys. Rev. Lett. 80, 1869 (1998).

[5] J. Arlt, P. Bance, S. Hopkins, J. Martin, S. Webster, A. Wilson, K. Zetie, and C.J. Foot, J. Phys. B 31, L321 (1998).

[6] D. Guéry-Odelin, J. Söding, P. Desbiolles, and J. Dalibard, Opt. Express 2, 323 (1998).

[7] D. Guéry-Odelin, J. Söding, P. Desbiolles, and J. Dalibard, Europhys. Lett. 44, 25 (1998).

[8] S.J.J.M.F. Kokkelmans, B.J. Verhaar, and K. Gibble, Phys. Ref. Lett. 81, 951 (1998).

[9] P.J. Leo, E. Tiesinga, P.S. Julienne, D.K. Walter, S. Kadlecek, and T.G. Walker, Phys. Ref. Lett. 81, 1389 (1998).

[10] V. Vuletic, A.J. Kerman, C. Chin, and S. Chu, Phys. Rev. Lett. 82, 1406 (1999).

[11] Yu.B. Ovchinnikov, I. Manek, and R. Grimm, Phys. Rev. Lett. 79, 2225 (1997).

[12] I. Manek, Yu.B. Ovchinnikov, and R. Grimm, Opt. Comm. 147, 67 (1998).

[13] A. Landragin, J.-Y. Courtois, G. Labeyrie, N. Vansteenkiste, C.I. Westbrook, and A. Aspect, Phys. Rev. Lett. 77, 1464 (1996).

[14] J. Söding, R. Grimm, and Yu.B. Ovchinnikov, Opt. Comm. 119, 652 (1995).

[15] I. Manek, doctoral thesis, Univ. Heidelberg 1999; MaxPlanck-Institut für Kernphysik report MPIH-V9-1999,
(1999).

[16] P. Desbiolles, M. Arndt, P. Szriftgiser, and J. Dalibard, Phys. Rev. A 54, 4292 (1996).

[17] A. Landragin, G. Labeyrie, C. Henkel, R. Kaiser, N. Vansteenkiste, C.I. Westbrook, and A. Aspect, Opt. Lett. 21, 1591 (1996).

[18] D. Boiron, A. Michaud, P. Lemonde, Y. Castin, C. Salomon, S. Weyers, K. Szymaniec, L. Cognet, and A. Clairon, Phys. Rev. A 53, R3734 (1996).

[19] D. Rychtarik, diploma thesis, Univ. Heidelberg 2000; Max-Planck-Institut für Kernphysik report MPI H - V 9, (2000).

[20] J. Weiner, V.S. Bagnato, S. Zilio, and P.S. Julienne, Rev. Mod. Phys. 71, 1 (1999).

[21] S. Bali, D. Hoffman, and T. Walker, Europhys. Lett. 27, 273 (1994).

[22] D. Hoffman, S. Bali, and T. Walker, Phys. Rev. A, 54, R1030 (1996).

[23] K. Burnett, P.S. Julienne, and K.-A. Suominen, Phys. Rev. Lett. 77, 1416 (1996).

[24] V. Vuletic, C. Chin, A.J. Kerman, and S. Chu, Phys. Rev. Lett. 83, 943 (1999).

[25] M. Hammes, diploma thesis, Univ. Heidelberg 1999; Max-Planck-Institut für Kernphysik report MPI H - V 24, (1999).

[26] W. Ketterle and N.J. van Druten, Adv. At. Mol. Opt. Phys. 37, 181 (1996).

[27] C.S. Adams, H.J. Lee, N. Davidson, M. Kasevich, and S. Chu, Phys. Rev. Lett. 74, 3577 (1995).

[28] H. Engler, T. Weber, M. Mudrich, R. Grimm, and M. Weidemüller, Phys. Rev. A, in press; preprint physics/0003075.

[29] For optical Zeeman pumping we use a weak circulary polarized beam resonant with the $F=3 \rightarrow F^{\prime}=3$ transition and aligned parallel to a static magnetic field 
of a few Gauss. In addition the usual GOST repumping light with mixed polarization provides hyperfine repumping via the $F=4 \rightarrow F^{\prime}=4$ transition. In this scheme the state of interest $\left(F=3, m_{F}=3\right)$ is the only dark state and thus efficiently populated. In a Hanle-type experiment [19] we have observed that the pumping beams lead to very weak heating in the presence of the longitudinal magnetic field. With a transverse magnetic field destroying the dark state, strong heating was observed. This confirms the predominant population of the dark state.

[30] D.M. Stamper-Kurn, H.-J. Miesner, A.P. Chikkatur, S. Inouye, J. Stenger, and W. Ketterle, Phys. Rev. Lett. 81, 2194 (1998).
[31] Yu.B. Ovchinnikov, S.V. Shul'ga and V.I. Balykin, J. Phys. B: At. Mol. Opt. Phys. 24, 3173 (1991).

[32] A.I. Safonov, S.A. Vasilyev, I.S. Yashnikov, I.I. Lukashevich, and S. Jaakkola, Phys. Rev. Lett. 81, 4545 (1998).

[33] P. Szriftgiser, D. Guéry-Odelin, M. Arndt, and J. Dalibard, Phys. Rev. Lett. 77, 4 (1996).

[34] R. Côté, B. Segev, and M.G. Raizen, Phys. Rev. A 58, 3999 (1998).

[35] R. Marani, L. Cognet, V. Savalli, N. Westbrook, C.I. Westbrook, and A. Aspect, Phys. Rev. A 61, 053402 (2000).

[36] F. Saif, B. Bialynicki-Birula, M. Fortunato, and W.P. Schleich, Phys. Rev. A 58, 4779 (1998). 